\title{
Matched Filtering of Numerical Relativity Templates of Spinning Binary Black Holes
}

\author{
Birjoo Vaishnav, Ian Hinder, and Frank Herrmann \\ Center for Gravitational Wave Physics, Penn State, University Park, PA 16802 \\ Deirdre Shoemaker \\ Center for Gravitational Wave Physics, Institute of Gravitational Physics and Geometry, \\ Department of Physics, Penn State, University Park, PA 16802
}

\begin{abstract}
Tremendous progress has been made towards the solution of the binary-black-hole problem in numerical relativity. The waveforms produced by numerical relativity will play a role in gravitational wave detection as either test-beds for analytic template banks or as template banks themselves. As the parameter space explored by numerical relativity expands, the importance of quantifying the effect that each parameter has on first the detection of gravitational waves and then the parameter estimation of their sources increases. In light of this, we present a study of equal-mass, spinning binary-black-hole evolutions through matched filtering techniques commonly used in data analysis. We study how the match between two numerical waveforms varies with numerical resolution, initial angular momentum of the black holes and the inclination angle between the source and the detector. This study is limited by the fact that the spinning black-hole-binaries are oriented axially and the waveforms only contain approximately two and a half orbits before merger. We find that for detection purposes, spinning black holes require the inclusion of the higher harmonics in addition to the dominant mode, a condition that becomes more important as the black-hole-spins increase. In addition, we conduct a preliminary investigation of how well a template of fixed spin and inclination angle can detect target templates of arbitrary spin and inclination for the axial case considered here.
\end{abstract}

\section{INTRODUCTION}

Initial LIGO has reached its design sensitivity [1] and, along with other ground-based detectors around the world, is taking science data. The matched filtering technique employed to search for gravitational wave signals from inspiraling and merging compact binaries is at its best when accurate representations of the signal are used. One of the best sources for early detection is the inspiral and merger of two black holes. The accurate waveforms of the binary black hole (BBH) signal come from two sources: post-Newtonian theory and numerical relativity. Waveforms from post-Newtonian theory are well understood during the inspiral phase of the binary evolution up to an as yet unknown point close to the final plunge of the black holes. With numerical relativity, we attempt to determine the point at which the approximation breaks down and to supply the waveform for the last orbit(s) and merger. Because black holes in the $30-300 M_{\odot}[2]$ mass range are expected to merge at frequencies that are in the most sensitive part of the LIGO frequency band, the computation of $\mathrm{BBH}$ waveforms has been a priority for the numerical relativity community. Fortunately, remarkable progress continues to be made in numerical relativity, especially in solving the last orbits and merger of binary black hole systems.

Since the breakthroughs in numerical relativity that allowed the evolution of BBHs through an orbit, merger and ringdown [3, 4, 4, 5], many groups have achieved similar success. The numerical relativity has now begun evolving many orbits [6, 7, 8, 9] and exploring the parameter space such as mass ratio and spins 10, 11, 12, 13, 14, 15, 16, 17, 18, 19, 20]. Each evolution of a BBH system requires large amounts of computational resources. The resource requirements for longer evolutions with unequal masses and spins are larger still. Numerical relativity efforts will benefit from a priori knowledge of where best to spend those resources to create an appropriate library of waveforms. Additionally, the cost of doing data analysis for a large template bank with many parameters is also computationally expensive. Knowledge of the sensitivity of the waveforms to the parameters will guide efforts in data analysis and numerical relativity. As the library of numerical relativity waveforms grows, we can determine how the waveforms will be best employed in the search for gravitational waves and the characterization of their sources. They may be used in conjunction with post-Newtonian waveforms through stitching, as templates for binary mergers, or to test the quality of template spaces built from post-Newtonian and/or approximate waveforms.

In 21, a comparison of the closeness between current gravitational wave search template banks and numerical relativity waveforms from non-spinning $\mathrm{BBHs}$ has been performed. The numerical relativity waveforms were used as target signals against which the template banks currently in use by LIGO to search for inspiral gravitational waves were tested. They found matches greater than 0.96 for many of the analytic and approximate template families at $10-120 M_{\odot}$. In [9], a phenomenological family of waveforms was proposed to model the coalescence of the BBHs using a hybrid method that combines analytical and numerical relativity waveforms. They achieve matches greater than 0.99 at $30 M_{\odot}-130 M_{\odot}$ for non-spinning BBHs.

In this paper, we conduct a first-step investigation into the ramifications on matched filtering of including spin in the $\mathrm{BBH}$ waveforms provided by numerical relativ- 
ity. The set of spinning BBH waveforms that we use are provided by the Penn State group and were published in [15] in addition to several non-spinning BBH waveforms. The spinning binaries are axial configurations with the initial black holes having spins of equal magnitude where one is aligned and one anti-aligned with the orbital angular momentum for a set of four spin parameters, $a=J / M^{2}=\{0.2,0.4,0.6,0.8\}$. The physical parameters of the waveforms are the numerical resolution, $\Delta$, and the initial angular momentum of the black holes in terms of the spin parameter, $a$. Because the configuration is axial, the intrinsic parameters of the waveforms do not include the angles between the spins and the axis of the orbital angular momentum. We also include the radiation modes given by $\ell$ and $m$ of the spin weighted $s=-2$, spherical harmonics, ${ }_{-2} Y_{\ell m}$, which are the inclination and azimuthal angles between the source and detector in the source frame. These waveforms contain approximately two and a half orbits. Because of this, we restrict the total mass range to $m>50 M_{\odot}$, set by the initial frequency of the evolution and our choice of how we enter the LIGO band.

Both numerical accuracy, e.g. truncation errors, and astrophysical accuracy, e.g. initial data choices, will play a role in determining the viability of numerical relativity $\mathrm{BBH}$ waveforms acting as templates. Requirements may be more stringent when characterizing the sources of gravitational waves; however, in this paper we focus on the use of numerical relativity waveforms as potential templates for detection, not for parameter estimation. Requirements for detection were first placed on waveforms from $\mathrm{BBHs}$ generated by numerical relativity in references [2, 22]. This early work preceded the successful solution of the BBH problem by many years, but acts as a guide for determining the constraint on numerical resolution accurate enough for data analysis purposes. A similar method was employed more recently in reference 23] in connection with BBH evolutions of equal-mass, non-spinning black holes over several orbits. Ref. [23], made a prediction of a maximum match that numerical waveforms will resolve. In addition, we did a preliminary study of the impact that numerical errors can have on the faithfulness of numerical waveforms using a Zerilli-based toy model in [24]. We now employ similar tests as [2] and [23] and verify the predicted behavior of the match with resolution for our BBH evolutions.

Often in numerical relativity, waveforms are extracted in terms of coefficients of ${ }_{-2} Y_{\ell m}$ the dominant mode being the quadrupole mode $(\ell=2,|m|=2)$. For compact binary inspiral searches, restricted post-Newtonian templates are commonly used for detection [25]. These templates include only the dominant harmonic in the amplitude while including as much information as possible in the phase since phasing is the more important issue in matched filtering. Corrected-amplitude templates have been considered in [26] and found to reduce the signalto-noise ratios for LIGO and add features to the detection and parameter estimation for Advanced LIGO [27, 28].
In [23], it was found that using the $\ell=2$ mode was was good enough for detecting gravitational waves of nonspinning, equal mass binaries. We include in our analysis the angle from the spherical harmonics, $\theta$ and $\phi$, where $\theta$ is related to the inclination angle of the binary with respect to the detector but in the source frame. We find that, in general, we will need to include higher modes to accurately represent spinning $\mathrm{BBH}$ waveforms. We also explore the sensitivity of the match with the spin parameter of the black-holes' initial angular momentum and how well a reduced template bank would do in matching with a target template of arbitrary spin and inclination.

The paper is organized as follows. In $₫$ II we explain the techniques we have employed in our $\mathrm{BBH}$ code and in our data analysis algorithms used in the rest of the paper. We present our results in $\$$ III including a description of the equal-mass, spinning and non-spinning evolutions that produced the waveforms under consideration including their spectra. We first assess the quality of our waveforms in terms of resolution through an analysis of the convergence properties of our BBH code in III A. We also present how the match statistic can reflect the convergence properties of our waveform in $\$ \amalg I B$. In $₫$ IIIC we present our study of the spinning templates in terms of varying inclination angle. Finally, in \$IID we look at using a specific numerical relativity waveform as the template and check its ability to match a target of unknown spin and inclination. We conclude in $₫ \mathrm{IV}$.

\section{TECHNIQUES}

\section{A. Numerical Relativity Techniques}

We solve the fully nonlinear Einstein equations in the $3+1$ form. Bowen-York 29] puncture [30] initial data representing the two black holes is specified on a spacelike slice $t=0$. This allows us to specify the initial mass, linear momentum, coordinate location and spin of each black hole. The BSSN [31, 32, 33] formulation of the Einstein equations in the $\chi$ form [5] is used to evolve this data forward in time. The gauge conditions are modified versions of the $1+\log$ lapse and $\Gamma$-driver shifts,

$$
\begin{aligned}
\partial_{0} \alpha & =-2 \alpha K, \\
\partial_{0} \beta^{i} & =3 / 4 B^{i}, \\
\partial_{0} B^{i} & =\partial_{0} \tilde{\Gamma}^{i}-\eta B^{i},
\end{aligned}
$$

where $\partial_{0}=\partial_{t}-\beta^{j} \partial_{j}$. We choose $\eta=2$ as in [34]. The advection term $\beta^{j} \partial_{j} \tilde{\Gamma}^{i}$ removes certain zero-speed modes of the system as analyzed in [35]. These gauge choices, with the exception of this advection term, were found to be important for long-term stable and accurate evolutions of head-on collisions without excision [36]. The puncture locations are determined by integrating the equation $\partial_{t} x^{i}(t)=\beta^{i}(x(t))$ [37].

The Weyl scalar $\Psi_{4}=R_{\alpha \beta \gamma \delta} n^{\alpha} \bar{m}^{\beta} n^{\gamma} \bar{m}^{\delta}$ measures outgoing gravitational radiation at large distances from the 
source. The orthonormal tetrad $\left\{n^{\alpha}, l^{\alpha}, m^{\alpha}, \bar{m}^{\alpha}\right\}$ is constructed from the spherical coordinate basis vectors as in [38]. We decompose $\Psi_{4}$ into ${ }_{-2} Y_{\ell m}$ on spheres of constant coordinate radius.

We use the Cactus infrastructure for parallelization, $\mathrm{I} / \mathrm{O}$ and parameter handling. The initial data is computed using the TwoPunctures [39] thorn, a spectral code which solves the momentum constraint for the conformal factor of our conformally flat spatial metric. We use the moving punctures approach without excision [4, 5] so the singularity is not treated specially, apart from ensuring that it does not lie on a grid point initially. The evolution is performed using our BSSN thorn which is automatically generated using the Kranc [40] code generation package. $\Psi_{4}$ is also computed using a Kranc-generated thorn. For mesh refinement we use Carpet [41], and our MoveIt infrastructure for specifying the grid structure based on the locations of the black holes.

The code uses finite differences, and all spatial derivatives are computed using centered fourth order stencils, apart from the shift advection terms which are computed using upwind, lop-sided, fourth order stencils. This gives increased accuracy over using centered stencils throughout. The evolution stencil thus has three points adjacent to any given point in all three directions. For time integration we use fourth order Runge-Kutta with a Courant factor $d t / d x^{i}=1 / 2$.

We use 9 levels of box-in-box mesh refinement, where the outermost (base) grid covers the domain $x^{i} \in$ $[-320,320]$. This allows the outer boundary to be causally disconnected from the computation of $\Psi_{4}$ at $r=30 m$ until well after the wave has passed. The next three grids cover the domains $x^{i} \in[-160,160],[-80,80]$, and $[-40,40]$. These grids are all fixed in place throughout the simulation. The remaining 5 levels generically contain two grids each centered on one of the black holes. These grids are cubical of half-side 10, 5, 2.5, 1.25 and 0.625 . Our moving grids infrastructure handles the case of the two grids on a given refinement level overlapping by replacing them with a single grid which is the smallest grid containing both of them. Each grid has half the grid spacing of its parent grid, and we typically refer to the resolution of the finest grid as $h_{f}$. This grid structure allows the extraction sphere at $r=30 m$ to not intersect any refinement boundaries.

We use Berger-Oliger mesh refinement as implemented by Carpet, and we refine in time as well as in space. The evolution on the refined grids requires a boundary condition provided by the coarse grid. This is implemented by adding additional points outside the refinement boundary, which are filled by interpolation from the coarser grid. Since the evolution stencil is of size 3, this boundary must consist of at least three points. Since the fine grid must be updated twice as frequently as the coarser grid, the interpolation is in time as well as space. As noted in [41], for evolution equations which are first order in time but second order in space, this scheme leads to instabilities. One solu- tion is to interpolate from the coarse grid only at the start of the Runge-Kutta sub-stepping algorithm, not at every sub-step. Since on each sub-step the boundary points are not computed, additional buffer points are required outside the finer grid. These points are also filled by interpolation at the start of the sub-stepping algorithm. The total number of boundary points required is stencil_size $\times$ time_integrator_sub-steps $=12$. We use fifth order interpolation in space, and second order interpolation in time. This lowers the overall accuracy expected from 4th to 2nd, but using higher order time interpolation is prohibitively expensive in terms of computational resources. We find that using 9 boundary points instead of 12 does not affect the results significantly, but it reduces the computational cost 34]. We expect that the second order error from the mesh refinement boundaries is small, and that at low resolutions the scheme will appear fourth order accurate.

The initial data parameters are given in Tab. If The non-spinning R1 parameters are taken from 42], based on the quasi-circular sequence found in [43, 44, 45]. The spinning runs are those reported in [15]. These runs consist of two equal-mass black holes whose initial spins are oriented such that one is aligned with the orbital angular momentum and the other is antialigned, and the magnitudes of the spins are equal. The runs are labeled as S0.05-S0.20 and have spin parameters $a=\{0.2,0.4,0.6,0.8\}$. The black holes are located at positions $(0, \pm y, 0)$, have linear momentum $(\mp P, 0,0)$, spin $(0,0, \pm S)$, and bare puncture masses $m_{ \pm}$. The irreduceable masses (measured from the areas of the apparent horizons) are $m_{1}=m_{2}=m / 2$ where $m=m_{1}+m_{2}$ is the total mass of the binary.

TABLE I: Initial data parameters

\begin{tabular}{lccccccc}
\hline Model & $y / m$ & $P / m$ & $a$ & $m_{+} / m$ & $m_{-} / m$ & $m_{\text {ADM }} / m$ & $J_{\text {ADM }} / m^{2}$ \\
\hline \hline R1 & 3.257 & 0.133 & 0 & 0.483 & 0.483 & 0.996 & 0.868 \\
S0.05 & 2.95 & 0.13983 & 0.2 & 0.4683 & 0.4685 & 0.98445 & 0.825 \\
S0.10 & 2.98 & 0.13842 & 0.4 & 0.4436 & 0.4438 & 0.98455 & 0.825 \\
S0.15 & 3.05 & 0.13547 & 0.6 & 0.3951 & 0.3953 & 0.98473 & 0.825 \\
S0.20 & 3.15 & 0.13095 & 0.8 & 0.2968 & 0.2970 & 0.98499 & 0.825 \\
\hline
\end{tabular}

\section{B. Data Analysis Techniques}

Our numerical waveforms are calculated during evolution in terms of the Newman-Penrose $\Psi_{4}$, where

$$
r m \Psi_{4}(t, \vec{r})=\sum_{l m}{ }_{-2} C_{l m}(t, r)_{-2} Y_{l m}(\theta, \phi),
$$

and $r$ is the extraction radius. The relationship between $\Psi_{4}$ and $h_{+}(t)$ and $h_{\times}(t)$ is given by

$$
\Psi_{4}(t)=\frac{d^{2}}{d t^{2}}\left(h_{+}(t)-i h_{\times}(t)\right) .
$$


To carry out the matched filtering analysis, we compute $\tilde{h}_{+}(f)$ and $\tilde{h}_{\times}(f)$ directly from the Fourier transform of the real part of $\Psi_{4}$ such that

$$
\tilde{h}_{+}(f)=\mathcal{F}\left(\operatorname{Re}\left(\Psi_{4}\right)\right)(f) /\left(-4 \pi^{2} f^{2}\right) .
$$

This avoids issues regarding integration constants that arise from the time domain integration of $\Psi_{4}$ [46]. Our initial data contains gravitational radiation which does not correspond to that present in an astrophysical situation. This radiates away and is visible in the waveform as an initial data pulse. We remove this pulse from each waveform.

Our analysis of the match of the numerical relativity waveforms will follow along the lines of the matched filtering procedure for detecting gravitational waves. That is, given two time domain waveforms $h_{1}(t)$ and $h_{2}(t)$, the scalar or inner product between these two functions is defined as

$$
\left\langle h_{1} \mid h_{2}\right\rangle=4 \operatorname{Re} \int_{f_{\min }}^{f_{\max }} \frac{\tilde{h}_{1}(f) \tilde{h}_{2}^{*}(f)}{S_{h}(f)} d f,
$$

where the domain $\left[f_{\min }, f_{\max }\right]$ is determined by the detector bandwidth and $\tilde{h}(f)$ stands for the Fourier transform of the respective time series. $S_{h}(f)$ denotes the noise spectrum for which we use the initial LIGO noise curve. The fact that our numerical waveforms only contain a few orbits before merger means that they do not span the entire LIGO frequency band. The initial orbital frequency of these runs varies depending on the value of the spin between approximately $0.016 / \mathrm{m}$ and $0.024 / \mathrm{m}$. The most stringent lower limit on $f_{\min }$ would be $0.024 / \mathrm{m}$. We impose the condition that the signal-to-noise ratio would be coming entirely from the domain spanned by our numerical waveforms such that $f_{\min }$ is calculated for each mass of the template. This fixes the lowest mass for which the templates could be useful to $50 M_{\odot}$.

The match statistic [47], is properly defined as the maximized overlap between the signal and the template, however, we will use it as a measure between two templates $h_{1}$ and $h_{2}$. There are two extrinsic parameters: the time of arrival of the signal, $t_{0}$, and the initial phase of the orbit when it enters the LIGO band, $\Phi$. First, consider the maximized overlap between templates defined with only maximization over time given by

$$
\begin{aligned}
O_{\max }\left[h_{1}, h_{2}\right] & \equiv \max _{t_{0}} O\left[h_{1}, h_{2}\right], \\
O\left[h_{1}, h_{2}\right] & \equiv \frac{\left\langle h_{1} \mid h_{2}\right\rangle}{\sqrt{\left\langle h_{1} \mid h_{1}\right\rangle\left\langle h_{2} \mid h_{2}\right\rangle}} .
\end{aligned}
$$

In Eq. (8), the maximization is to be understood as the maximum overlap between $h_{1}$ and $h_{2}$ obtained by shifting the template in time, i.e. $h(t) \rightarrow h\left(t+t_{0}\right)$ leading to the numerator being written in frequency domain as

$$
\max _{t_{0}}\left\langle h_{1} \mid h_{2}\right\rangle=\max _{t_{0}} 4 \operatorname{Re} \int_{f_{\min }}^{f_{\max }} \frac{\tilde{h}_{1}(f) \tilde{h}_{2}^{*}(f) e^{2 \pi i f t_{0}}}{S_{h}(f)} d f .
$$

The Fourier transform is replaced by a discrete fast Fourier transform, transforming the integral into a discrete sum. When computing the match without phase optimization, we will typically compute the match of the templates using only $h_{+}$.

We also compute the match with an optimization over the phase, $\Phi$ of the template in addition to $t_{0}$. A waveform of arbitrary initial phase $\Phi$ is written as

$$
\tilde{h}(f)=\tilde{h}_{+}(f) \cos \Phi+\tilde{h}_{\times}(f) \sin \Phi .
$$

Given an arbitrary waveform, $\tilde{h}_{1}$, the phase optimization over the template, $\tilde{h}_{2}$, can be carried out using the normalized templates $e_{i,+, \times}=\tilde{h}_{i+, \times} /\left\|\tilde{h}_{i+, \times}\right\|$ where $i$ runs over 1,2 . In reference to the match we will always be referring to frequency domain templates and for ease of notation we shall refer to $\tilde{h}$ as $h$ in the match formulae. The typical match [48] is given by

$$
\begin{aligned}
M_{\mathrm{typ}} & \equiv \max _{t_{0}} \max _{\Phi_{2}} O\left[h_{1+}, h_{2}\right] \\
& \approx \max _{t_{0}} \sqrt{O\left[e_{1+}, e_{2+}\right]^{2}+O\left[e_{1+}, e_{2 \times}\right]^{2}},
\end{aligned}
$$

where we have assumed that $e_{2+}$ and $e_{2 \times}$ are nearly orthogonal, i.e. $\left\langle e_{2+} \mid e_{2 \times}\right\rangle \approx 0$. This approximation is valid only for angles near $(\theta=0, \phi=0)$ where the contribution from the modes other than $\ell=|m|=2$ is zero because of the spherical harmonic values. For a general waveform at nonzero $\theta$ and $\phi$, the overlap of the corresponding $h_{+}$and $h_{\times}$is of the order of a few percent. In our spinning waveforms, we find a maximum deviation from orthogonality of $\sim 3 \%$ at an angle that approaches $\pi / 2$. In order to avoid uncertainties in the matches at large angles, we construct an orthonormal waveform basis as done by [48, 49] and outlined below.

Any arbitrary polarization other than $(+, \times)$ can be expressed as a linear combination of these linearly independent basis vectors. The phase optimization of the match can then be done in terms of the new orthonormal basis vectors, one of which we choose to be $e_{i+}$ and the other $e_{i \perp}$ given as

$$
e_{i \perp}=\left(e_{i \times}-e_{i+}\left\langle e_{i+} \mid e_{i \times}\right\rangle\right)\left(1-\left\langle e_{i+} \mid e_{i \times}\right\rangle^{2}\right)^{-1 / 2} .
$$

One can see that $\left\langle e_{i+} \mid e_{i \perp}\right\rangle=0$ by construction. Given such orthonormalized basis vectors for two sets of parameters $i=1,2$, we can calculate the upper and lower bounds on the phase optimized match [50].

In this new orthonormal basis, we rewrite the typical match from Eq. (12) by matching the + polarization of one template while optimizing over the phase of the other. This mimics the situation in which one of the waveforms acts as the template and can be maximized over its phase, $\Phi_{2}$, while keeping the phase of the second template, $\Phi_{1}$, fixed, as follows

$$
\begin{aligned}
M_{\mathrm{typ}} & \equiv \max _{t_{0}} \max _{\Phi_{2}} O\left[h_{1+}, h_{2}\right] \\
& =\max _{t_{0}} \sqrt{O\left[e_{1+}, e_{2+}\right]^{2}+O\left[e_{1+}, e_{2 \perp}\right]^{2}} .
\end{aligned}
$$


In general, the phase of the signal could take any value, and one would like to know the best and worst possible values of the match. The expression for the best match (the upper bound) is given by [50]

$$
\begin{aligned}
M_{\text {best }} & \equiv \max _{t_{0}} \max _{\Phi_{1}} \max _{\Phi_{2}} O\left[h_{1}, h_{2}\right] \\
& =\max _{t_{0}}\left[\frac{A+B}{2}+\left[\left(\frac{A-B}{2}\right)^{2}+C^{2}\right]^{\frac{1}{2}}\right]^{\frac{1}{2}}
\end{aligned}
$$

in which the phases of each template are optimized. The minimax match is given by the case when one maximizes the phase of one of the templates but minimizes over the other. This is to mimic the worst case scenario when the signal phase is such that it gives lower matches even when maximized over the template phase, given by [50]

$$
\begin{aligned}
M_{\operatorname{minimax}} & \equiv \max _{t_{0}} \min _{\Phi_{2}} \max _{\Phi_{1}} O\left[h_{1}, h_{2}\right] \\
& =\max _{t_{0}}\left[\frac{A+B}{2}-\left[\left(\frac{A-B}{2}\right)^{2}+C^{2}\right]^{\frac{1}{2}}\right]^{\frac{1}{2}}
\end{aligned}
$$

and for both cases the functionals $A, B, C$ are written in terms of the orthonormal basis functions $\left(e_{1+}, e_{1 \perp}\right)$ and $\left(e_{2+}, e_{2 \perp}\right)$ corresponding to the two templates being compared

$$
\begin{aligned}
& A \equiv\left\langle e_{1+} \mid e_{2+}\right\rangle^{2}+\left\langle e_{1+} \mid e_{2 \perp}\right\rangle^{2}, \\
& B \equiv\left\langle e_{1 \perp} \mid e_{2+}\right\rangle^{2}+\left\langle e_{1 \perp} \mid e_{2 \perp}\right\rangle^{2}, \\
& C \equiv\left\langle e_{1+} \mid e_{2+}\right\rangle\left\langle e_{1 \perp} \mid e_{2+}\right\rangle+\left\langle e_{1+} \mid e_{2 \perp}\right\rangle\left\langle e_{1 \perp} \mid e_{2 \perp}\right\rangle .
\end{aligned}
$$

Note the symmetry of the formulae upon interchange of the two templates which arises from the fact that only the relative phase between the two templates should matter. Because of this, which template is maximized and which minimized is interchangeable. We will refer to the target's phase being minimized and the template's phase being maximized since this makes sense in a detection scenario.

\section{RESULTS}

We present our results in three categories: the variation of the match with resolution, including convergence tests of the waveforms; the variation of the match with inclination and spin; and how well we can differentiate between a target of unknown inclination angle and spin in the mass range of $50 M_{\odot}$ to $300 M_{\odot}$ given a template of fixed parameters. The spectra of the BBH waveforms from the models R1 and S0.05-S0.20 are shown in Fig. 1. in which we plot $|h(f)|$. The $\theta=0$ lines correspond to the $\ell=|m|=2$ mode. The final black hole in all these runs settled to the same final spin with a spin parameter $a \approx 0.66$.

In Fig. 1, we can see that when $\theta=0$, the spectra for each value of $a$ become very similar for $f>0.05 m$. This

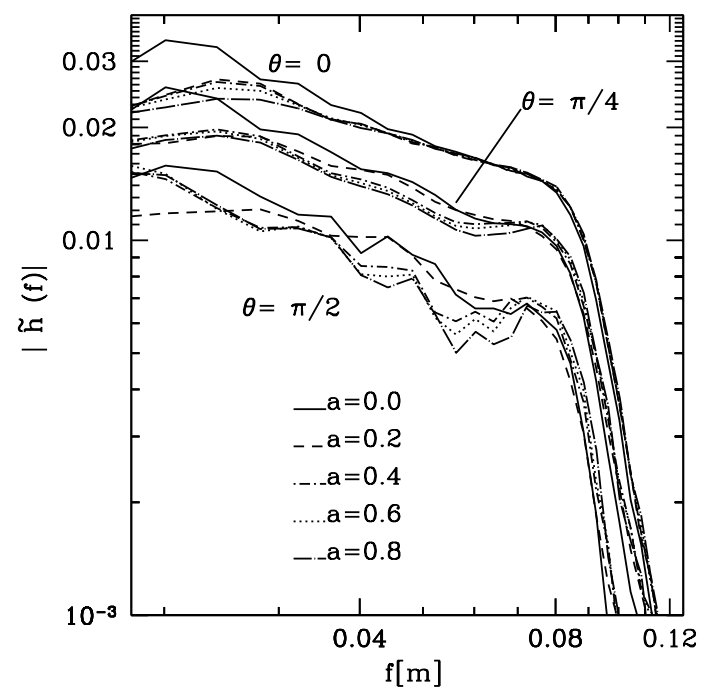

FIG. 1: We plot the spectrum of the wave versus frequency of waveforms from different initial spins in a log-log plot. The spinning cases all have resolutions of $m / 40$ and the nonspinning, $m / 38.4$. We present three cases of inclination angle, $\theta$, for each set of spins, and the units are $f_{\text {code }}=f_{\text {phys }} / m$.

is because all of the configurations we evolved settled down to very similar final black holes. When $\theta$ deviates from zero, however, we can already see from the spectra that we get more variation between the different spinning waveforms. This is in part due to the larger resolution requirements for the higher harmonics, however, as we shall see in the following results, the resolution differences do not account for all of the variation.

Firstly, in $\oiiint$ IIA we investigate the convergence of the dominant, $\ell=|m|=2$, mode for non-spinning BBHs, the convergence of the spinning waveforms was published in [15] to be between third and fourth order. In $₫$ IIIB we investigate the quality of the numerical waveforms as templates in matched filtering in terms of the resolution including spin and inclination angle. Next, in $\amalg I I C$ we show how the inclusion of modes $\ell<5$ affects the match calculations when compared to just using the dominant mode waveform $\ell=|m|=2$ as a function of the spin. Additionally, we determine the effect of using a finite extraction radius on the quality of the matches. Lastly, in $₫ \mathrm{IID}$, we compare the different spin waveforms to each other, including the dependence on $\theta$ but keep the masses the same. This is equivalent to checking the faithfulness [50] of the waveforms assuming the orientation of the source for the different sources to be the same. While this assumption will need to be relaxed in a fully general treatment for data analysis, it suffices to demonstrate the importance and impact of using higher than dominant modes in the analysis of these merger waveforms. 


\section{A. Numerical Convergence}

A convergence test of the spinning series of runs called S0.05-S0.20 is given in [15]. Here we investigate of the quality of our numerical waveforms with a convergence test of the equal-mass, non-spinning R1 series of runs. These runs are names R1a-R1f and only differ in their resolution. The suffix corresponds to the grid spacing of the finest grid surrounding each black hole. The grid spacings are $\Delta_{a-f}=$ $\{m / 25, m / 32, m / 38.4, m / 44.8, m / 51.2, m / 57.6\}$ We consider the convergence properties of the $\ell=m=2$ mode of $\Psi_{4}$ computed on the coordinate sphere at $r=30$. In Fig. 3 we plot $\operatorname{Re}\left[\Psi_{4}^{2,2}\right]$, and Fig. 2 shows that $\Psi_{4}^{2,2}$ computed at $r=30$ appears to converge monotonically with resolution to a continuum solution, and that the high resolution results agree well with each other. Similar results (not shown) are obtained for $\arg \Psi_{4}^{2,2}$ and the coordinate locations of the black holes. Using the runs R1a, R1b, and R1f we demonstrate fourth order convergence of the amplitude and phase of $\Psi_{4}^{2,2}$ in Fig. 4 and Fig. 5. Were we to plot only the highest resolution runs, the fourth order convergence would be lost; i.e. there is a source of error which spoils the convergence at high resolutions. However, as can be seen from Fig. 2, the waveforms still appear to converge to a continuum solution, just at a different order.

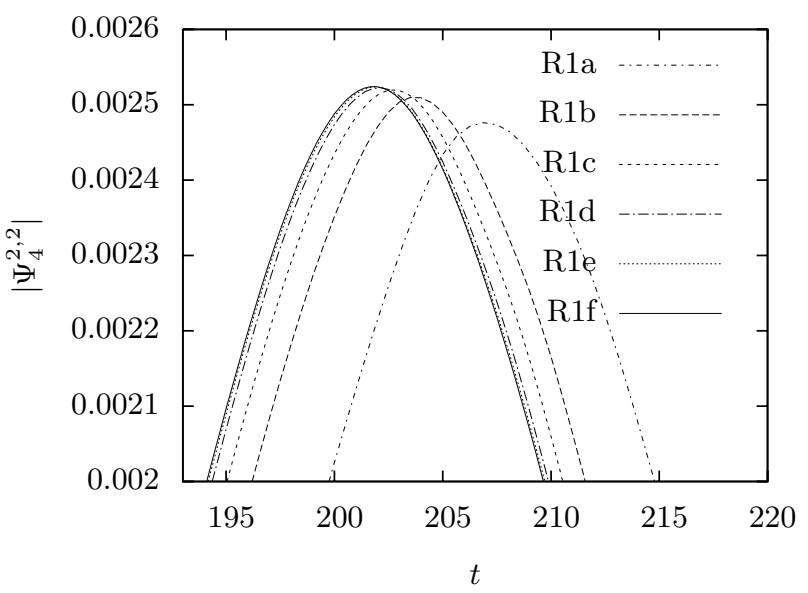

FIG. 2: Monotonic change in $\left|\Psi_{4}^{2,2}\right|$ as resolution is increased. It appears that the function is converging to a continuum solution.

\section{B. Variation of Templates with Resolution}

We can follow the numerical convergence properties through matched filtering since we know how the numerical codes approximate the exact solution to the partial differential equations discretely. For finite differencing

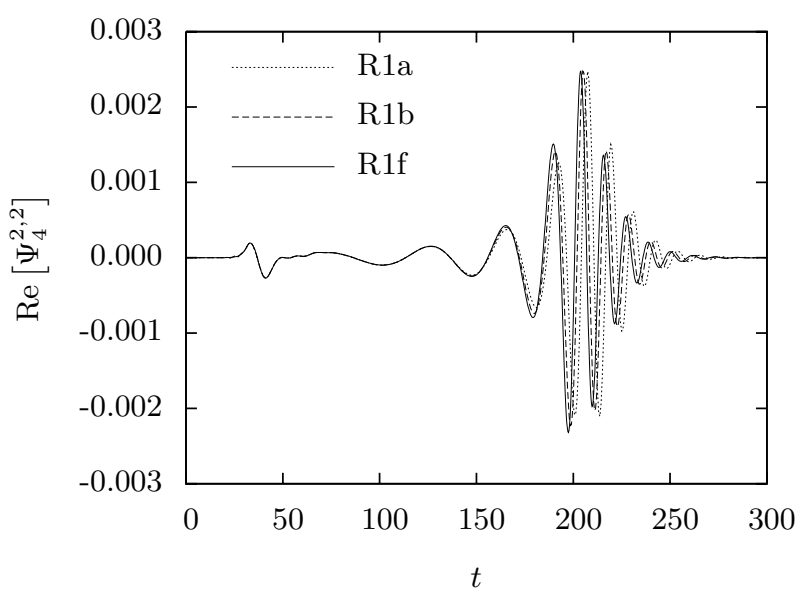

FIG. 3: $\operatorname{Re}\left[\Psi_{4}^{2,2}\right]$ computed at $r=30$ for three different resolutions. The feature at $t=40$ is due to the gravitational radiation present in the initial data, and will be cut out of the waveform before taking Fourier transforms for data analysis.

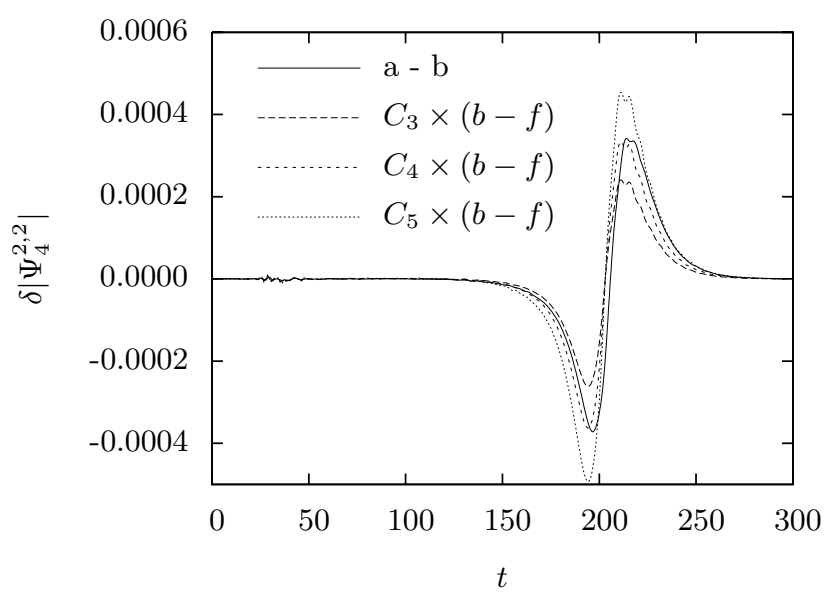

FIG. 4: Convergence of $\left|\Psi_{4}^{2,2}\right|$ using the highest and the two lowest resolutions. $C_{3}, C_{4}, C_{5}$ are the scaling factors expected for third, fourth and fifth order convergence respectively. We see that $a-b$ matches most closely with $C_{4} \times(b-f)$ indicating fourth order convergence.

this is expressed as

$$
h(t)=h_{0}(t)+c(t) \Delta^{p}+\mathcal{O}\left(\Delta^{p+1}\right)
$$

where $h_{0}(t)$ is the exact solution found when the resolution goes to infinity, $\Delta$ is the grid spacing $(\Delta=\Delta x=$ $\Delta y=\Delta z \propto \Delta t), p$ is the order of the truncation error and $c(t)$ is a time dependent scaling independent of the grid spacing. Since our code is approximately fourth order accurate, we expect to scale with $p=4$. One can calculate $h_{0}(t)$ using Richardson extrapolation of three discrete, convergent solutions of different resolution. We have computed $h_{0}(t)$ for the R1 series of runs using R1a, $\mathrm{R} 1 \mathrm{~b}$, and R1f and found that $h_{0}(t)$ coincides to within 


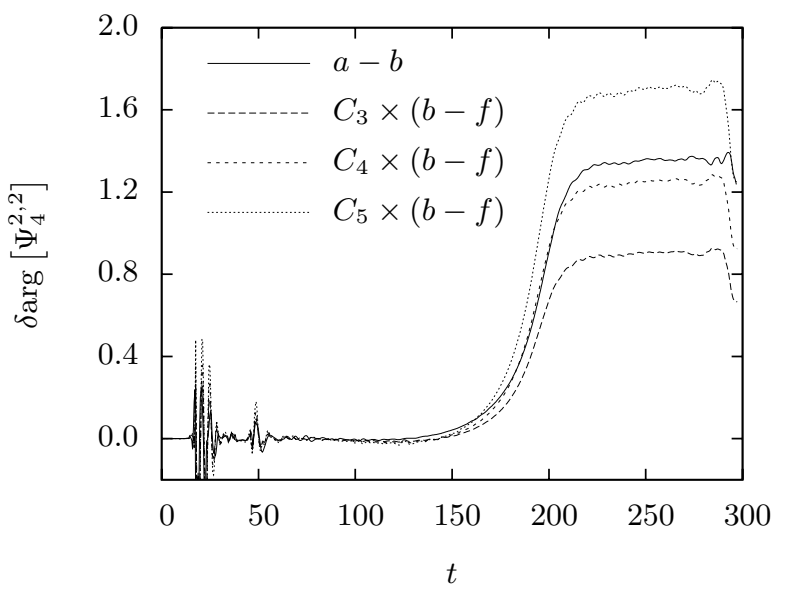

FIG. 5: Convergence of $\arg \Psi_{4}^{2,2}$. As in Fig. 4, we see fourth order convergence.

$10^{-5}$ in amplitude with the waveform evolved with the finest resolution, $\Delta_{f}=m / 57.6$. In light of this, we will use our finest resolution run, indicated as $h\left(\Delta_{f}\right)$, in place of the Fourier transform of $h_{0}(t)$.

We can predict the behavior of the maximized overlap as a function of resolution between two waveforms that differ only in their resolution by expanding the match equation, Eq.(8), about $\Delta^{p}=0$. Note that when $\Delta^{p}$ is a constant in time, we can express Eq.(17), in the Fourier space as $h_{i}=h_{0}+c \Delta_{i}^{p}$, where $i$ is running over each waveform. We expand the match for the case of two templates at two different resolutions to be as general as possible, i.e. $O\left[h_{1}\left(\Delta_{i}\right), h_{2}\left(\Delta_{j}\right)\right]$ as follows

$$
\begin{aligned}
O\left[h_{i}, h_{j}\right] & =1-\frac{1}{2}\left(\Delta_{i}^{p}-\Delta_{j}^{p}\right)^{2}\left(\frac{\langle c \mid c\rangle}{\left\langle h_{0} \mid h_{0}\right\rangle}-\left(\frac{\left\langle c \mid h_{0}\right\rangle^{2}}{\left\langle h_{0} \mid h_{0}\right\rangle^{2}}\right)\right) \\
& +\mathcal{O}^{(3)}\left(\Delta^{p}\right) .
\end{aligned}
$$

The above equation was expanded for two matches of different resolution but the match with the Richardson extrapolated solution can be recovered by setting $\Delta_{j}=0$ up to the order expressed. This equation indicates that the mismatch, $\left(1-O_{\max }\right)$, goes like $\Delta^{2 p}$. Fig. (6) shows the dependence of the $O_{\max }$ on the resolution for three choices of the total mass, $m=\{50,100,200\} M_{\odot}$. The least squares fit is done for several possible values of $\Delta^{2 p}$ including $p=3,4,5$. The best fit is found for $p=4$, and is the fit pictured in Fig.(6).

Because of the computational cost of generating solutions to the Einstein equations at three resolutions for every waveform of interest, Flanagan and Hughes 22] suggested computing the match between templates of neighboring resolutions, such as $O_{\max }\left[h\left(\Delta_{a}\right), h\left(\Delta_{b}\right)\right]$ for example. A variation, used by Baumgarte et al [23], is to compute the series of matches as $O_{\max }\left[h\left(\Delta_{i}\right), h\left(\Delta_{f}\right)\right]$, where the first template runs over all the resolutions available, denoted by $\Delta_{i}$, and the second template's resolution is fixed to the finest available.

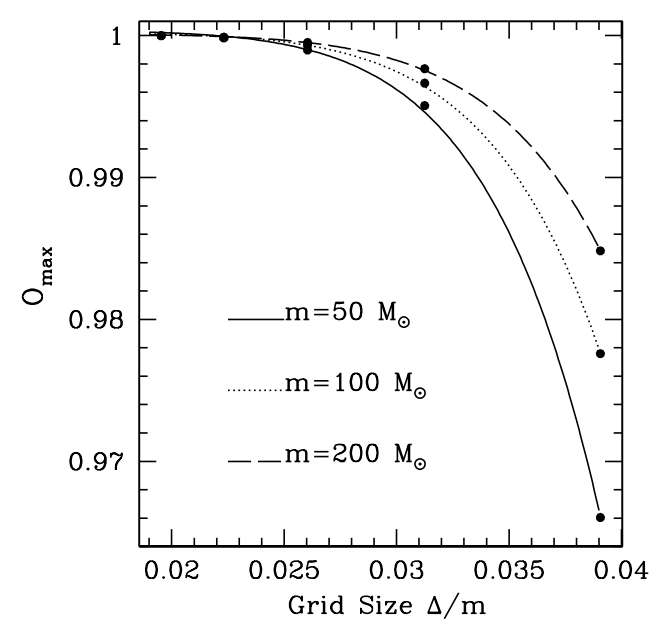

FIG. 6: The maximized overlap, $O_{\max }\left[h_{i}, h_{f}\right]$, from Eq. (8) is plotted as a function of grid size for three total masses indicating fourth order convergence. The data are given by the points and the least square fitting by the lines. The $p=4$ fit differs for different masses because of the weighting by the current LIGO noise curve $S_{h}(f)$.

We plot the Flanagan and Hughes type of overlaps, $O_{\max }\left[h_{+}\left(\Delta_{a}\right), h_{+}\left(\Delta_{b}\right)\right]$ and $O_{\max }\left[h_{+}\left(\Delta_{b}\right), h_{+}\left(\Delta_{c}\right)\right]$ and three cases where we vary the resolution of one template while keeping the second template fixed to the finest resolution, $O_{\max }\left[h_{+}\left(\Delta_{i}\right), h_{+}\left(\Delta_{f}\right)\right]$ where $i=a, b, c$, in Fig. (7). These matches are unoptimized over the phase, but optimized over the time $t_{0}$ and follow the definition given in Eq. (8).

The threshold of match above which the resolutions will be "good enough" for detection is typically set to 0.98 . Clearly from the figure we can conclude for the $\theta=$ 0 , a resolution of $\Delta_{b}=m / 32.0$ was enough for the entire mass range to reach a minimum match of 0.98 and that, for a lower resolution of $\Delta_{a}=m / 25.6$, we only achieved $O_{\max }>0.98$ for total masses larger than $100 M_{\odot}$. One of the main features of the matches with resolution is the decrease in the match toward smaller masses. This is partly due to the low number of gravitational wave cycles we have present in the waveforms. The numerical errors, however, will increase as we increase the total number of cycles so the choice of resolution will depend on the number of orbits evolved. As we evolve more cycles, we will also be extending the total mass range into smaller masses.

We now have to choose which match to use in assessing the quality of the templates. The matches are expected to get closer to one when phase optimization is included. Since the phase of a given signal waveform depends on the detector orientation and several such variables, it could differ from the template up to a constant phase factor and the match would vary depending on what the phase is. The worst case would occur when the arbitrary phase is 


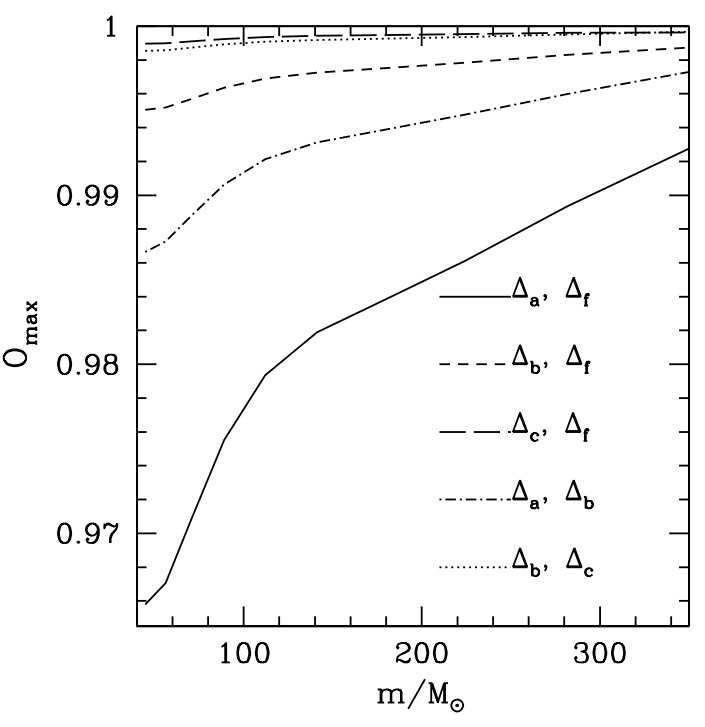

FIG. 7: The maximized overlaps, $O_{\max }\left[h_{1+}, h_{2+}\right]$, are plotted as a function of mass for several resolutions of our equalmass BBH series of runs. Labels $(a, b, c)$ stand for the coarse runs $R 1 a, b, c$ with resolutions $m / 25.6, m / 32.0, m / 38.4$, while $R 1 f$ has resolution $m / 57.6$. The overlap between adjacent resolutions $O_{\max }\left[h_{+}\left(\Delta_{a}\right), h_{+}\left(\Delta_{b}\right)\right.$ has deceptively high values compared to the matches with the finest resolution.

such that there is maximum destructive interference between the target and the template, and sets up the lower bound on the possible matches, $M_{\text {minimax }}$. If one does not add any phase factor to the numerically generated template, one gets what is called the typical match and if the phase factor is chosen for maximum constructive interference, it yields the "best" match, $M_{\text {best }}$. The value of $M_{\text {best }}$ is a measure of the closest distance between two templates. We'll use the minimax match for the rest of the paper to report our results in terms of lower bounds and use the typical and best matches to illustrate the range over the different type of matches.

To demonstrate the differences in the match with choice of optimization, we tabulate examples of optimization in Tab. III for few selected total masses between the coarsest and finest runs. No optimization at all is listed in the second column, optimization over just $t_{0}$ from Eq. (8) in the third, the typical match Eq. (14) in the fourth, minimax, Eq. (17), in the fifth and finally best match, Eq. (16), in the final column. The phase optimized matches, the last three columns, exceed the the threshold of $O_{\max }>0.98$ for even the least resolved waveform when matched with the finest resolution waveform.

We also calculated the minimax matches, see Eq. (17), for all the $R 1$ resolutions, although we do not plot them here, and found that for all the resolutions $M_{\operatorname{minimax}} \geq$ 0.99 in the mass range under consideration and show the same variation as the overlap. These results hold only
TABLE II: Comparing matches with different optimizations between the coarsest $(R 1 a)$ and the finest $(R 1 f)$ runs for selected total masses. The second column is the normalized overlap between the two waveforms without optimization over $t_{0}$, the third column is the match with optimization over $t_{0}$, the fourth is the typical match, the fifth the minimax, and the final column is the best match.

\begin{tabular}{cccccc}
\hline$m$ & $O$ & $O_{\max }$ & $M_{\text {typ }}$ & $M_{\operatorname{minimax}}$ & $M_{\text {best }}$ \\
\hline \hline 44.67 & 0.7987 & 0.9658 & 0.9979 & 0.9974 & 0.9983 \\
70.79 & 0.5458 & 0.9709 & 0.9989 & 0.9989 & 0.9991 \\
112.2 & 0.2308 & 0.9794 & 0.9994 & 0.9993 & 0.9995 \\
177.8 & 0.04864 & 0.9837 & 0.9994 & 0.9993 & 0.9996 \\
281.8 & -0.06719 & 0.9893 & 0.9993 & 0.9993 & 0.9996 \\
\hline
\end{tabular}

for $\theta=0$ and $a=0$.

We now analyze the resolution needs of the spinning $\mathrm{BBH}$ waveforms of which we only have three resolutions for each of the spinning configurations in contrast to the five available for the non-spinning case. These are labeled $\Delta_{\text {coarse }}=m / 32, \Delta_{\text {med }}=m / 38.4$, and $\Delta_{\text {fine }}=m / 40$. Later in this paper, we find that it is necessary to include the higher modes for the spinning $\mathrm{BBH}$ templates; and, therefore, as we analyze the spinning $\mathrm{BBH}$ waveforms versus resolution we also include its variation with $\theta$. At inclination angle $\theta=0$ only the $\ell=|m|=2$ mode is present in $h$, and when $\theta \neq 0$ all the modes get mixed. We do not include modes $\ell \geq 5$ in constructing $h$ because these were too small to be well resolved for the evolutions we use in this paper. The variation of $\theta$ is presented for two suggestive cases, $\theta=\pi / 4$ and $\theta \sim \pi / 2$. Values of $\theta$ are not taken to be exactly on the plane since the radiation is then linearly polarized and cannot be described with two basis vectors and cannot be maximized over the phase at that point, but instead at $\theta \sim \pi / 2=89 \pi / 180$.

For the $\theta=0$ case, all the spinning waveforms have a $M_{\text {minimax }} \geq 0.99$ over the entire mass range for matches between the $\Delta_{\text {coarse }}$ and $\Delta_{\text {fine }}$ and between $\Delta_{\text {med }}$ and $\Delta_{\text {fine. }}$. These matches also follow the same trend with mass, i.e. decreasing matches with decreasing total mass, as reported in Tab. II. For $\theta \neq 0$, we plot the minimax match for the $a=0.8$ spin case between both the highest and coarsest and highest and medium resolved waveforms at the two values of $\theta$ in Fig. 8 . We only show the $a=0.8$ waveforms since the matches of the high and low resolution of the other spin and non-spinning runs are all $M_{\text {minimax }} \geq 0.99$ at these angles again over the entire mass range. Note, however, that even though the matches are very high, they decrease with increasing spin. This is expected since we are keeping the number of points across the black hole fixed, but as the spin increases, the horizon area decreases, hence the effective numerical resolution of a high initial spin waveform is lower than that of a low initial spin waveform. The case of 
$\theta \sim \pi / 2$ gave us the lowest matches of any angles between 0 and $\pi / 2$ and shows that the minimax match of the $\Delta_{\text {fine }}$ and $\Delta_{\text {med }}$ resolutions is $M_{\operatorname{minimax}} \geq 0.97$. While the match of the coarsest resolution run at higher masses is $M_{\text {minimax }} \sim 0.92$, which indicates that the coarse resolution is not good enough for meeting the threshold when $a=0.8$ and $\theta \sim \pi / 2$. For the rest of the paper, we will use the finest resolution for all spin cases to ensure that the errors due to resolution are no greater $3 \%$ in the most difficult case to resolve: the high spin, high angle case, and should be much lesser in other cases.

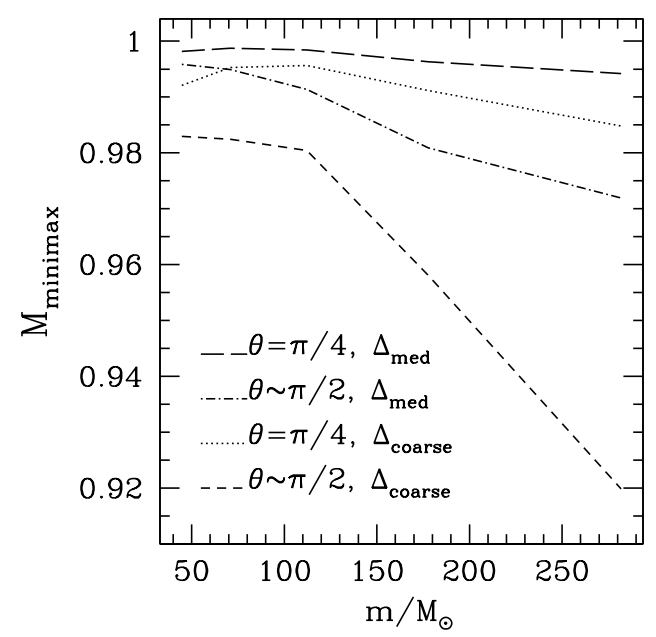

FIG. 8: We plot the minimax match between the waveforms of $a=0.8$ for two cases of inclination angle, $\theta=\pi / 4$ and $\theta=\pi / 2$ and two different resolutions $\Delta_{\text {fine }}$ with $\Delta_{\text {med }}$ and $\Delta_{\text {fine }}$ with $\Delta_{\text {coarse }}$. Corresponding matches for lower spins are all $\geq 0.99$ and are not shown here.

Fig. 8 shows two features of the minimax match, namely it decreases with increasing $\theta$ and with increasing $m$. The decrease of the match in Fig. 8 at higher mass is in contrast to Fig. 7 There are two differences between the figures, one is the phase optimization which, from Tab. [II, we can see does not change the trend of the match versus mass. The second difference is the inclusion of higher modes. The overall match will be reduced with non-zero $\theta$ since the frequencies of the modes increase with $\ell$, placing stronger resolution requirements. The highest spin we analyze, $a=0.8$, has the lowest minimax matches of all the spin cases, as low as $M_{\text {minimax }}=0.92$ at the largest angle. Large spins demand more resolution in general and this is seen particularly when resolving modes with $\ell>2$. For the lower spin runs, $a \leq 0.6$, the waveforms are well resolved and the matches are all $\geq 0.99$ over the entire mass range even for $\theta \sim \pi / 2$. The decrease in match is seen most strongly at the larger mass range since this mass range targets the merger and ringdown part of the signal. We expect these match values and all the matches reported in this section to change for waveforms with more gravitational wave cycles since the numerical errors will grow as the length of the run increases and the accumulating phase errors will likely play a larger role.

\section{The Variation of Spinning Templates with Inclination Angle}

An observer on the orbital axis of a merging binary, at $\theta=\phi=0$, will see a fully circularly polarized waveform where $\ell=|m|=2$ is the only nonzero mode. An observer, however, in the orbital plane of the binary or the equatorial plane of the final black hole will see linearly polarized radiation with the contribution from $\ell=|m|=2$ at its minimum. All other observers with intermediate orientations would see elliptically polarized waves that are combinations of all the modes. In practice, numerical relativity can only resolve a finite set of modes. Since the orientation of any given binary will be unknown $a$. priori, we analyze the templates' dependence on the inclination and azimuthal angles. The location of the detector in the center of mass frame fixes the mode content and the orientation of the detector fixes the initial phase of the waveform.

The resolution will now be fixed to the finest for the spin cases and the corresponding resolution for the nonspinning case. We use the minimax match as defined in Eq. (17), to see how different the $\ell=|m|=2$ waveforms, given by $h(\theta=0, \phi=0)$, are from the full waveforms, $h(\theta, \phi)$, for a given $a$. Our focus is on $\theta$ since it causes a larger variation in the match than $\phi$. To reduce the number of parameters considered, and we set $\phi=\pi / 2$ for the rest of the paper when $\theta \neq 0$. If we were to relax this condition, the minimax matches would vary on the order of a percent. To observe the variation with $\theta$, we first fix the total mass for both templates, $h_{1}$ and $h_{2}$, to $100 M_{\odot}$. In Fig. 9 we present the minimax matches as a function of $a$ for templates at $\theta=\{0, \pi / 4, \pi / 3, \pi / 2\}$. By holding the target template to $\theta=0$ for each spin case, we can determine at what angle and spin the matches drop below the threshold. In the case of $\theta=0$, the templates have the same parameters and $M_{\operatorname{minimax}}=1$ as it should. For $a=0$, all the templates match the $\theta=0$ target within $M_{\text {minimax }}=0.98$. The plot also indicates that $\theta=0$ is close to the full waveform for $\theta \leq \pi / 3$ for all of the spin cases. For $\theta>\pi / 3$, however, the match drops below 0.98 for $a>0.2$. As the inclination with the axis increases, the higher modes become important with increasing spin.

To study inclination variation as a function of mass, we add the scale with total mass coming from the LIGO noise curve. We limit the presentation to two spins, $a=$ 0.2 and $a=0.8$ for clarity. The best, minimax and typical matches are plotted as a function of $m$ in Fig. 10 and Fig. 11. The matches are between $h_{1}(\theta=0)$ and $h_{2}\left(\theta_{i}\right)$ for three inclination angles each $\theta_{i}=\pi / 4, \pi / 3, \sim \pi / 2$. We plot all three matches to demonstrate the range that the matches can take depending on the choice of phase 


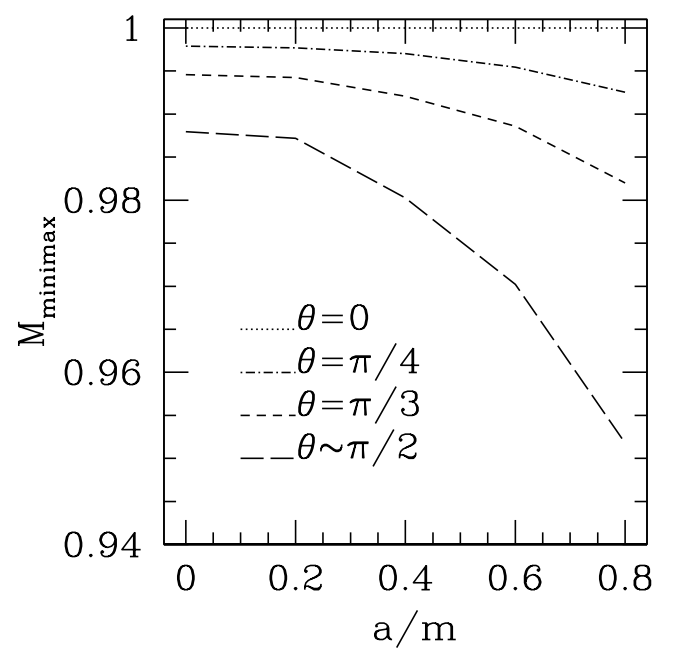

FIG. 9: The minimax match, $\left.M_{\operatorname{minimax}}\left[h_{1}(\theta=0)\right], h_{2}\left(\theta_{i}\right)\right]$, vs $a$ is plotted for the values $\theta_{i}=\{0, \pi / 4, \pi / 3, \pi / 2\}$ for the case $m=100 M_{\odot}$.

optimization. The typical match is given by the lines and the minimax and best matches are specified as the lower and upper error bars respectively. One can see that the minimax match between the two templates sets a lower bound on the phase optimized matches. The minimax match dips below 0.98 for $\theta>\pi / 3$ and $m>100_{\odot}$ for both spin cases; and, unfortunately, dips below 0.98 for the entire mass range for $\theta \sim \pi / 2$ when $a=0.8$.

An even more stringent test is the best match. If the best match of a given angle waveform is smaller than some threshold then we would know that the higher modes are significant and need to be used in creating the template bank. We note that for both spin cases the best match is below 0.98 for $\theta \sim \pi / 2$ at masses greater than $170 M_{\odot}$ indicating the need to include higher modes to make a detection at large inclination and high masses.

One can see from the plots that the lower bound of the match between the circularly polarized and the highly elliptically polarized waveform is lower in the high mass cases. The decrease in the match at larger total masses may be indicating that the ringdown is more sensitive to the presence of higher modes than the merger itself, since the deviation from 0.98 is larger than expected from numerical error alone. These figures also suggest that the $\ell=|m|=2$ mode is closer to the full waveform at lower masses and lower spins. The fraction of events lost by not including the higher modes needs to be calculated to predict the full impact.

The waveforms that we are using have been extracted from the numerical solution of the Einstein equation at a finite radius. In order to assess how the extraction radius, $r_{\text {ext }}$, affects the waveforms at a large extraction radius, we tabulate the $M_{\text {minimax }}$ of the $a=0.8$ waveforms in Tab. III], The matches are computed for a template fixed at $\theta=0$ and the target template at four values of $\theta$ to

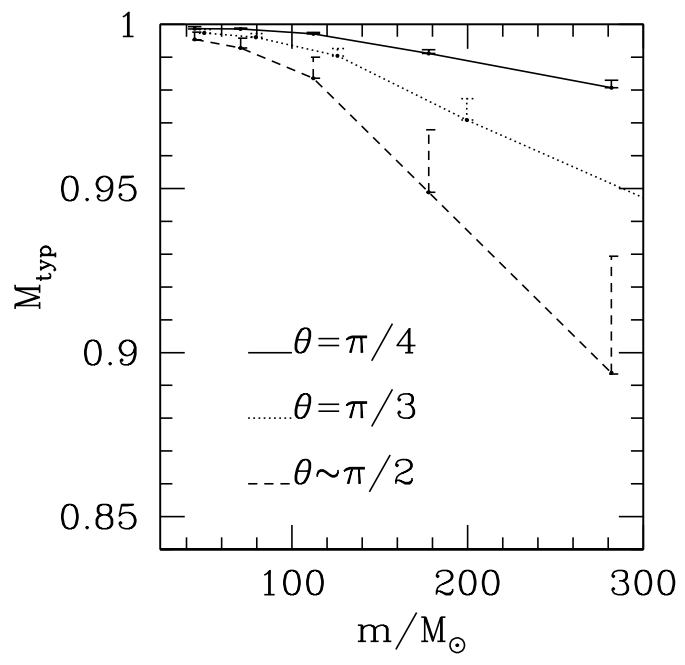

FIG. 10: The range of phase optimized matches between the $\theta=0$ waveform and the full waveform for a low spin case, $a=$ 0.2 . The curves denote typical match $M_{\mathrm{typ}}\left[h_{1}(\theta=0), h_{2+}\left(\theta_{i}\right)\right]$ for $\theta_{i}=\{\pi / 4, \pi / 3, \sim \pi / 2\}$. The lower end of the error bar is given by the minimax match and the higher end is given by the best match. The phase optimization here is done over the phase of the $h_{1}$ template.

measure how much of the variation with $\theta$ depends on $r_{\text {ext }}$. The variation with the extraction radius is of the order of $\sim 1.3 \%$ in the worst case. There is an interplay between better dynamical quality and increased numerical error as $r_{\text {ext }}$ is increased, which is more noticeable in modes higher than $\ell=2$. For the rest of the paper we use the waveforms at $r_{\text {ext }}=30 \mathrm{~m}$.

TABLE III: Minimax matches for $m=100 M_{\odot}$ and $a=0.8$ between two waveforms at the same extraction radius but different inclination angle given by $M_{\operatorname{minimax}}\left[h_{1}(\theta=0), h_{2}\left(\theta_{2}\right)\right]$.

\begin{tabular}{ccccc}
\hline$r_{\text {ext }}$ & $\theta_{2}=0$ & $\theta_{2}=\pi / 4$ & $\theta_{2}=\pi / 3$ & $\theta_{2} \sim \pi / 2$ \\
\hline \hline 30 & 0.9779 & 0.9602 & 0.9538 & 0.9518 \\
40 & 0.9773 & 0.9530 & 0.9465 & 0.9519 \\
50 & 0.9756 & 0.9473 & 0.9423 & 0.9546 \\
60 & 0.9734 & 0.9445 & 0.9409 & 0.9564 \\
\hline
\end{tabular}

\section{Comparing the Various Spin Configuration Waveforms}

Given the computational cost of producing templates for BBHs, we investigate how well a reduced template matches a target template of arbitrary spin and inclination angle at a fixed resolution $(\Delta=m / 40)$. We will study several cases, including spin configurations relative to each other at different inclination angles for a 


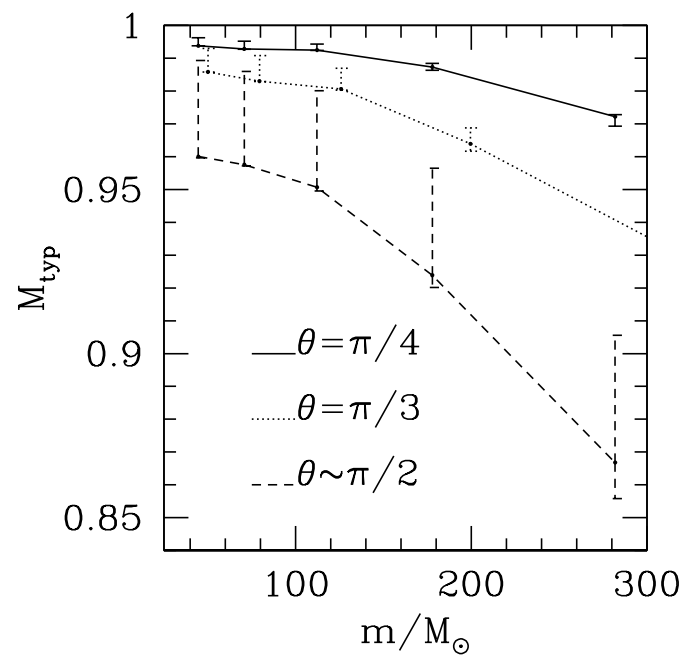

FIG. 11: The range of phase optimized matches between the $\theta=0$ waveform and the full waveform for a high spin case, $a=$ 0.8 . The curves denote typical match $M_{\text {typ }}\left[h_{1}(\theta=0), h_{2+}\left(\theta_{i}\right)\right]$ for $\theta_{i}=\{\pi / 4, \pi / 3, \sim \pi / 2\}$. The lower end of the error bar is given by the minimax match and the higher end is given by the best match. The phase optimization here is done over the phase of the $h_{1}$ template.

fixed mass of $100 M \odot$ and then we specialize to a few angles and study the typical, minimax and the best phase optimized matches.

The simplest approach to compare the spinning waveforms is to fix $\theta=0$ for both the target and template. We then choose the template to have some spin and calculate the match of that template with a target that varies with $a$, such that we calculate $M_{\text {minimax }}\left[h_{1}\left(a_{i}\right), h_{2}\left(a_{j}\right)\right]$ where $a_{i}$ and $a_{j}$ run over all the combinations of spin. The minimax matches between all combinations of the spins are $\geq 0.995$ over the mass range considered. This reflects the fact that the binaries approach the same final black hole and that the initial spin gets radiated away in modes other than $\ell=|m|=2$, where they are almost identical during the late stages of the binary merger (sometimes called universality [42]). This would mean that we could not distinguish between the non-spinning and spinning waveforms making the non-spinning case sufficient for a detection template bank but potentially making parameter estimation problematic. This degeneracy with spin, however, does not hold when we include more radiation modes.

We explore the matches between different spin templates at different inclination angles, considering first the templates with a fixed mass of $100 M_{\odot}$. The variation of the minimax match between a template of $a=0$ and target templates that vary with $a$ is presented in Fig. 12 and with both the template and target varying with $a \neq 0$ in Fig. 13. In terms of the match, Fig. 12 corresponds to a minimax match, $M_{\operatorname{minimax}}\left[h_{1}(a=0), h_{2}\left(a_{i}\right)\right]$, and Fig. 13 between six combinations of the four spinning configura- tions, $M_{\operatorname{minimax}}\left[h_{1}\left(a_{i}\right), h_{2}\left(a_{j}\right)\right]$ both versus $\theta$. In both figures, at $\theta \leq \pi / 3$ the template is within a $M_{\operatorname{minimax}}$ of 0.98 with the targets, making them indistinguishable. When the template is fixed at $a=0$ in Fig.12 the match is $>0.98$ for all $\theta$ except with $a>0.6$, which shows a stronger drop-off with angle for higher initial spins. Fig. 13 shows that the matches between adjacent templates among the $a=\{0.2,0.4,0.6,0.8\}$ group are better than 0.99 , while the matches that have a spin parameter difference $a_{1}-a_{2} \geq 0.4$ drop below 0.98 at higher angles. These templates are based on short waveforms of just two and a half cycles and the matches will likely get worse when more cycles are included. We conclude from these plots that the $\ell=|m|=2$ mode continues to dominate the other modes at small angles, $\theta<\pi / 4$, leading to similar matches between a spinning and non-spinning configuration. As the inclination angle grows, however, the presence of higher modes becomes more pronounced, breaking the degeneracy between the waveforms from different initial spin configurations.

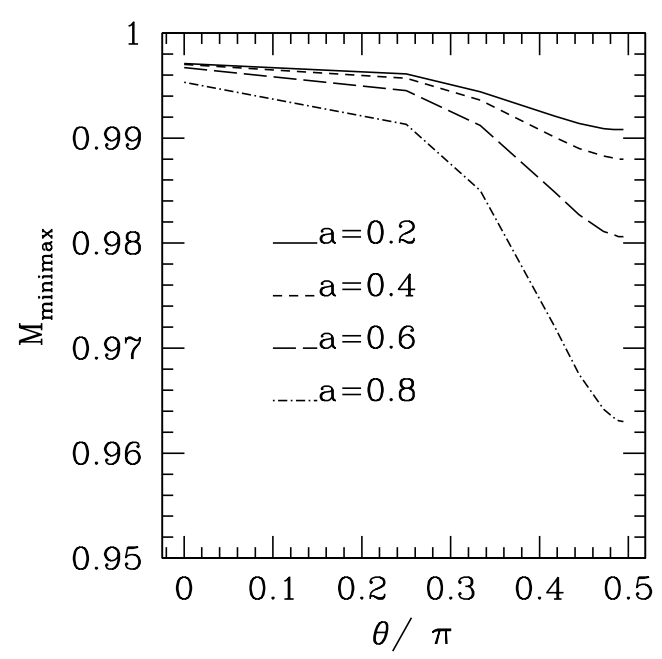

FIG. 12: The minimax match $M_{\text {minimax }}\left[h_{1}(a=0), h_{2}\left(a_{i}\right)\right]$ as a function of $\theta$ between the waveforms of different spins at the same resolution $m / 40$, with the non-spinning waveform at resolution $m / 38.4$. The mass of the final black hole for all the cases is $100 M_{\odot}$ and the spins run over $a_{i}=\{0.2,0.4,0.6,0.8\}$. Note the monotonic decrease of the match with angle.

For completeness, we present the table of the range of values over which the phase optimized matches of the different spinning waveforms can vary in Tab. IV] These matches are evaluated at $\theta \sim \pi / 2$ to report the widest range of matches calculated. The table indicates that the range of matches is higher at lower masses and tends to decrease with mass.

To place the minimax matches between the spins in context with the typical and the best matches, we present the typical match $M_{\text {typ }}\left[h_{+1}(a=0), h_{2}\left(a=a_{j}\right)\right]$ for $a_{j}=$ $\{0.2,0.8\}$ and $\theta=\{\pi / 2, \pi / 4\}$ in Fig. [14. The error bars are given by the minimax and the best match in order 


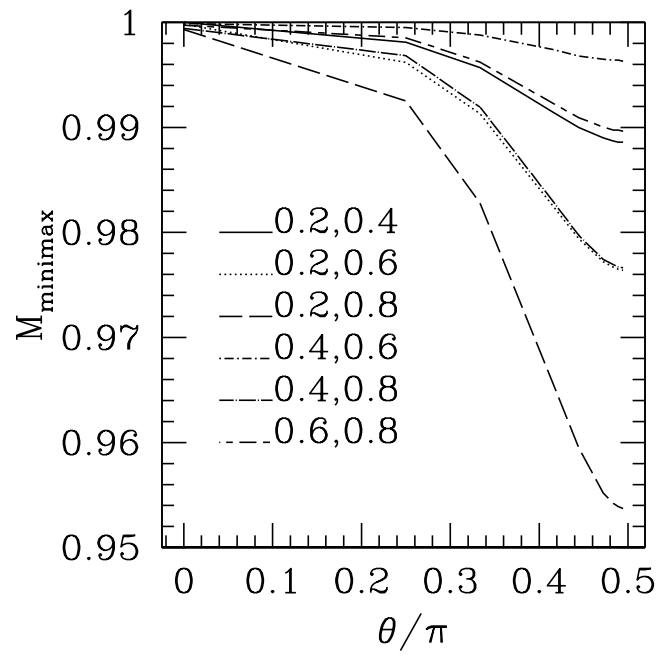

FIG. 13: The minimax match between the waveforms of different nonzero initial spins, $M_{\operatorname{minimax}}\left[h_{1}\left(a_{i}\right), h_{2}\left(a_{j}\right)\right]$ as a function of $\theta$. The mass of the final black hole for all the cases is $100 M_{\odot}$ and $\left\{a_{i}, a_{j}\right\}$ runs over $\{0.2,0.4,0.6,0.8\}$. Note the monotonic decrease of the minimax match with the angle $\theta$.

TABLE IV: The range of phase optimized matches reported as $\left(M_{\text {minimax }}, M_{\text {best }}\right)$ between two templates of various spin pairs $\left(a_{1}, a_{2}\right)$ where both templates have $\theta \sim \pi / 2$.

\begin{tabular}{cccc}
\hline$\left(a_{1}, a_{2}\right)$ & $50 M_{\odot}$ & $120 M_{\odot}$ & $300 M_{\odot}$ \\
\hline \hline$(0.0,0.2)$ & $(0.8940,0.9513)$ & $(0.9228,0.9618)$ & $(0.9446,0.9701)$ \\
$(0.0,0.4)$ & $(0.8570,0.9507)$ & $(0.8882,0.9604)$ & $(0.9141,0.9684)$ \\
$(0.0,0.6)$ & $(0.8595,0.9530)$ & $(0.8898,0.9618)$ & $(0.8234,0.9427)$ \\
$(0.0,0.8)$ & $(0.7325,0.9196)$ & $(0.7896,0.9326)$ & $(0.8369,0.9449)$ \\
$(0.2,0.4)$ & $(0.9860,0.9964)$ & $(0.9884,0.9967)$ & $(0.9858,0.9954)$ \\
$(0.2,0.6)$ & $(0.9742,0.9927)$ & $(0.9751,0.9927)$, & $(0.9665,0.9912)$ \\
$(0.2,0.8)$ & $(0.9526,0.9832)$ & $(0.9497,0.9793)$, & $(0.9194,0.9681)$ \\
$(0.4,0.6)$ & $(0.9973,0.9993)$ & $(0.9959,0.9988)$, & $(0.9945,0.9971)$ \\
$(0.4,0.8)$ & $(0.9808,0.9956)$ & $(0.9732,0.9902)$, & $(0.9611,0.9738)$ \\
$(0.6,0.8)$ & $(0.9911,0.9982)$ & $(0.9880,0.9950)$, & $(0.9824,0.9867)$ \\
\hline \hline
\end{tabular}

to demonstrate their variation with mass. As seen in Fig. 14, the difference between the minimax and best matches is slight at small values of $\theta$ and $a$, while for large angles it can vary over the order of $\sim 3 \%$. The variation is much higher at lower masses because there are more cycles in the sensitive part of the LIGO noise curve making the match more sensitive to the relative phasing of the templates. The figure indicates that using a non-spinning waveform as the template in detection would cause the largest losses when the target has large spin and angle.

We have seen the variation of the match between tem-

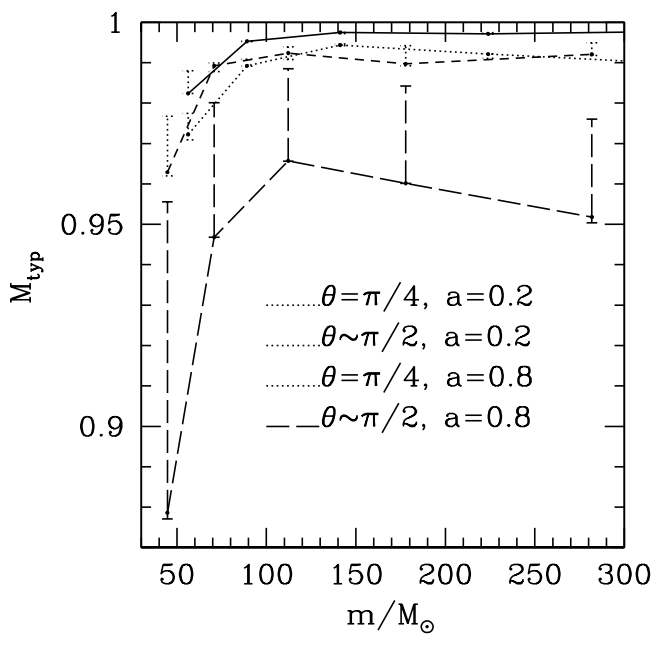

FIG. 14: We plot the range and the typical values of phase optimized match between the waveforms of different initial spins, $M_{\text {typ }}\left[h_{+1}\left(a=0, \theta_{j}\right), h_{2}\left(a_{i}, \theta_{j}\right)\right]$ as a function of mass where $a_{i}=\{0.2,0.8\}$ and $\theta_{j}=\{\pi / 4, \pi / 2\}$.

plates of the same spin parameters and different angles and different spin parameters and the same angle. In setting up a template bank using these numerical waveforms, it may be useful to know if just a couple of templates at some specific inclination and spins can cover the whole set of waveforms considered here.

For this study we calculate the minimax matches between a template of a given value of spin and inclination angle and a target with a spin parameter varying over $a_{i}=\{0.0,0.2,0.4,0.6,0.8\}$ and a fixed, but different inclination angle. In Fig. 15 we plot the variation of the minimax match for a template with $a_{1}=0.0$ and $\theta_{1}=\pi / 4$ with target templates of varying spin and $\theta_{2} \sim \pi / 2$ as a function of total mass. We made the choice of the template to have $\theta_{1}=\pi / 4$ since we have concluded that a choice of $\theta=0$ is going to fail to match well over a range of target inclination angles, especially at lower masses. We choose the target template to have $\theta_{2} \sim \pi / 2$ to calculate the worse case scenario for the inclination angle of the target. In Fig. 16 we substitute a template of $a_{1}=0.4$ and keep all the other parameters the same as in Fig. 15. This improves the matches at smaller total mass. One can see from the figures that the higher the match threshold, the smaller the mass range covered by the template. A coarse template bank would do better with $a=0.4$ and $\theta=\pi / 4$ than $a=0$ at the same $\theta=\pi / 4$ especially for the lower masses and hints that there may be an optimal way to lay out this template space. Another feature is that for higher masses, the matches for both the cases look similar, as the late-merger/ringdown has some universality-independence from the initial spin.

The use of these axial-spin waveforms as templates for detection has some limitations. The waveforms investigated had the angle between the initial spins kept fixed, 


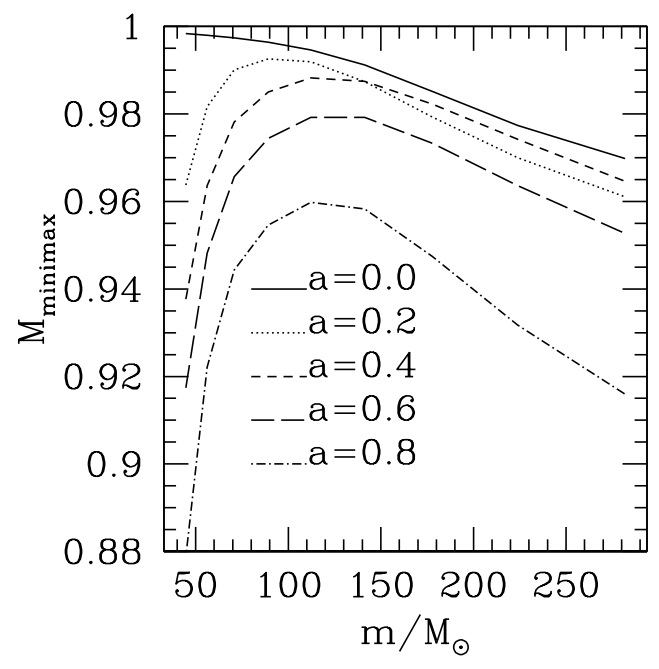

FIG. 15: We plot the minimax match between the target templates of different initial spins at a fixed $\theta$ with a template of $a=0.0, M_{\operatorname{minimax}}\left[h_{1}(a=0, \theta=\pi / 4), h_{2}\left(a_{i}, \theta=\pi / 2\right)\right]$ as a function of mass. Notice the low matches at the smaller masses.

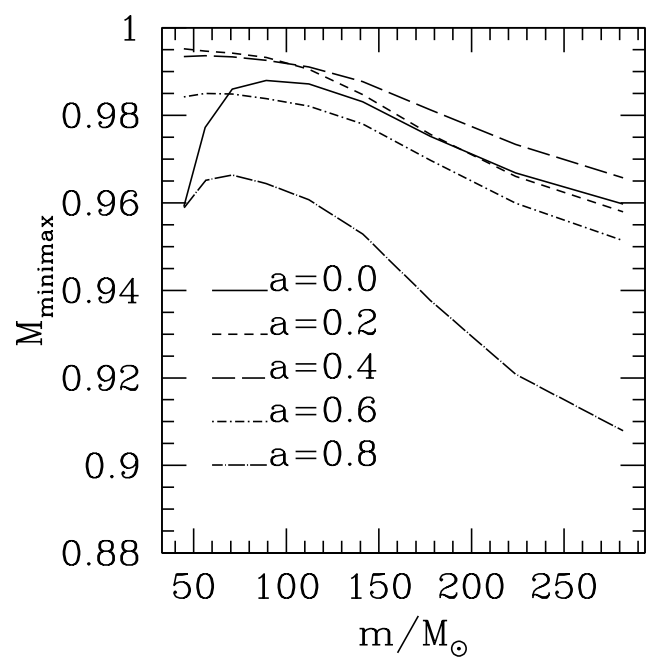

FIG. 16: We plot the minimax match between the target templates of different initial spins at a fixed $\theta$ with a template of $a=0.4, M_{\text {minimax }}\left[h_{1}(a=0.4, \theta=\pi / 4), h_{2}\left(a_{i}, \theta=\pi / 2\right)\right]$ as a function of mass.

and it is not clear how the conclusions we draw from this study hold as this condition is relaxed. Although it is likely that the higher radiation modes will play an even bigger role than they do for the relatively simple case investigated here. The major limitation however, is the small number of gravitational wave cycles present in the waveforms. The ISCO frequency is about $0.02 / \mathrm{m}$. The mass at which the ISCO frequency hits the seismic wall of LIGO noise curve of $40 \mathrm{~Hz}$ is about $100 M_{\odot}$. If one allows the lower limit in the match integrals to vary or be fixed at $0.02 / m=70 \mathrm{~Hz}$ for example, then we can trust these matches up to $\approx 60 M_{\odot}$ assuming that we will search for events that are merging in the LIGO band with little to no inspiral. Although the results will not change too much at high masses even if inspiral is included in the numerical templates, low mass results are expected to have lower matches. Finally, another limitation of these matches is that it assumes identical total mass for the template and the waveform being compared to it. This assumption needs to be relaxed to estimate the "effectualness" of templates for detection, which will be done in a future work.

\section{CONCLUSION}

The parameter space of the $\mathrm{BBH}$ system is rapidly being explored by the numerical relativity community. Given how much time and effort in takes to evolve the $\mathrm{BBH}$ spacetimes and then to use those waveforms in detection schemes, it is becoming increasingly important to probe the parameter space of the $\mathrm{BBH}$ system as the waveforms become available. With this motivation, we have investigated a series of waveforms from the final two to three orbits of a binary-black-hole system made up of equal but spinning black holes with matched filtering. Although the waveforms we use are not long enough in terms of gravitational wave cycles to cover the entire LIGO frequency band, they shed light on what weight different parameters will have in the matched filtering procedure using LIGO's initial noise curve. We study the dependence of the match on the intrinsic parameters of numerical resolution, inclination and spin as well as the extrinsic parameters of arrival time and phase.

Given the numerical convergence properties of our waveforms, we first predict and show that the match has a dependence on the resolution of $\Delta^{2 p}$, in which a fourth order evolution scheme has $p=4$. For the resolutions of our $\mathrm{BBH}$ evolutions, we get very high matches $(>0.98)$ for resolutions above $m / 32$ when $a=0$ and above $m / 38$ for waveforms with $a=0.8$. The resolutions are only appropriate for the cases we have investigated. For the waveforms generated out of the $\ell=|m|=2$ mode, we find that the match values between the same waveforms at different resolution decreases as the total mass decreases. We only report matches from $50 M_{\odot}-300 M_{\odot}$, where $50 M_{\odot}$ is set by our initial orbital velocity.

We then include the variation of the match with the inclination angle. It is often convenient and instructive to extract the gravitational waveform, $\Psi_{4}$, decomposed into spin-weighted spherical harmonics as a function of $\ell$ and $m$. Including modes higher than the dominant mode, $\ell=|m|=2$, is equivalent to allowing the inclination angle of the binary system to vary. Only at $\theta=0$ would the $\ell=|m|=2$ mode be the only harmonic in the waveform. The target waveform will have an unknown orientation; and, therefore we vary the inclination to study what effect this may have on detection. As we include all the modes 
in the analysis the match decreases, to as low as $\approx 0.85$, must notably for larger masses and higher spins. Some decrease in match is expected since the higher modes require more resolution; however, the decrease was greater than that accounted for by the resolution alone.

Finally, in light of the computational effort involved in searching over large, densely populated template banks, we calculate the match between waveforms of different initial spin to see if a reduced set of spinning waveforms would be good enough. The waveforms we use in this analysis evolve toward the same Kerr black hole $(a \approx 0.66)$ even though the initial spins of the individual black holes vary from 0.0 to 0.8 because of the fixed initial ADM angular momentum. This results in very high matches, $M_{\text {minimax }}>0.99$, between all the spinning and non-spinning waveforms for the dominant mode. Without adding information from the higher radiation modes, all the spinning $\mathrm{BBH}$ waveforms appeared the same in terms of matched filtering.

Once $h$ was constructed using modes $\ell<5$, this degeneracy broke. We constructed two "typical" cases, one in which the template had a fixed $\theta=\pi / 2$ and $a=0$ and the other $a=0.4$. The templates were matched against a target template also at $\theta=\pi / 2$ for each spinning waveforms. When $a=0$, the matches were as low as 0.88 versus $m$, but were $>0.95$ for $m>100 M_{\odot}$. By choosing an intermediate spin of $a=0.4$, the matches were improved for the lower masses, $m<100 M_{\odot}$, increasing the lowest value from 0.88 to 0.96 . We speculate that this indicates that there may be an optimal layout for the template space for a coarse search over spins. The matches were always at their highest at $100 M_{\odot}$, although not necessarily greater than 0.98 .

In summary, we have investigated the sensitivity of matched filtering to the presence of spin and radiation modes in a limited case of spinning BBH waveforms. We find that if we ignore the modes higher than $\ell=2$, there is very little difference between the spinning waveforms, especially at large $m$ due to our choice of initial data. We do find, however, that the inclusion of larger $\ell$ changes this picture in two ways. Two templates with the same spin but different inclination only match for low values of the inclination angle and two templates of the same inclination angle but different spin only match when the spins are within 0.2 of each other. Matching well means that $M_{\operatorname{minimax}} \geq 0.98$. In general, the higher modes reduce the matches more at large masses than at small masses. It remains to be seen how these results change when the spins are no longer fixed to axial configurations, but more elaborate initial data will likely vary more with $\ell$.

\section{ACKNOWLEDGMENTS}

We thank Shane Larson, Sam Finn, Pablo Laguna and Ben Owen for their insightful discussions on topics in this paper. We thank The Center for Gravitational Wave Physics is supported by the NSF under cooperative agreement PHY 01-14375. Work partially supported by NSF grant PHY-0354821 to DS.
[1] LIGO Scientific Collaboration and TAMA Collaboration, Phys. Rev. D 73, 102002 (2006).

[2] E. Flanagan and S. Hughes, Phys. Rev. D 57, 4535 (1998).

[3] F. Pretorius, Phys. Rev. Lett. 95, 121101 (2005), grqc/0507014.

[4] J. G. Baker, J. Centrella, D. Choi, M. Koppitz, and J. van Meter, Phys. Rev. Lett. 96, 111102 (2006).

[5] M. Campanelli, C. O. Lousto, P. Marronetti, and Y. Zlochower, Phys. Rev. Lett. 96, 111101 (2006).

[6] J. G. Baker, S. T. McWilliams, J. R. van Meter, J. Centrella, D.-I. Choi, B. J. Kelly, and M. Koppitz, grqc/0612117 (2006).

[7] A. Buonanno, G. B. Cook, and F. Pretorius, grqc/0610122 (2006)

[8] H. P. Pfeiffer, D. A. Brown, L. E. Kidder, L. Lindblom, G. Lovelace, and M. A. Scheel, gr-qc/0702106 (2007).

[9] P. Ajith, S. Babak, Y. Chen, M. Hewitson, B. Krishnan, J. T. Whelan, B. Bruegmann, P. Diener, J. Gonzalez, M. Hannam, et al., arXiv.org:0704.3764 (2007).

[10] F. Herrmann, I. Hinder, D. Shoemaker, and P. Laguna, gr-qc/0601026 (2006).

[11] J. A. Gonzalez, U. Sperhake, B. Bruegmann, M. Hannam, and S. Husa, Phys. Rev. Lett. 98, 091101 (2007).

[12] M. Campanelli, C. O. Lousto, and Y. Zlochower, Phys. Rev. D 74, 041501 (2006).
[13] M. Campanelli, C. O. Lousto, and Y. Zlochower, Phys. Rev. D 74, 084023 (2006).

[14] M. Campanelli, C. O. Lousto, Y. Zlochower, B. Krishnan, and D. Merritt, gr-qc/0612076 (2006).

[15] F. Herrmann, I. Hinder, D. Shoemaker, P. Laguna, and R. A. Matzner, gr-qc/0701143 (2007).

[16] M. Koppitz, D. Pollney, C. Reisswig, L. Rezzolla, J. Thornburg, P. Diener, and E. Schnetter, grqc/0701163 (2007).

[17] J. A. Gonzalez, M. D. Hannam, U. Sperhake, B. Brugmann, and S. Husa, gr-qc/0702052 (2007).

[18] M. Campanelli, C. O. Lousto, Y. Zlochower, and D. Merritt, Ap. J. 659, L5 (2007).

[19] M. Campanelli, C. O. Lousto, Y. Zlochower, and D. Merritt, gr-qc/0702133 (2007).

[20] W. Tichy and P. Marronetti, gr-qc/0703075 (2007).

[21] Y. Pan, A. Buonanno, J. G. Baker, J. Centrella, B. J. Kelly, S. T. McWilliams, F. Pretorius, and J. R. van Meter, arXiv:0704.1964 (2007).

[22] E. Flanagan and S. Hughes, Phys. Rev. D 57, 4566 (1998).

[23] T. Baumgarte, P. Brady, J. Creighton, L. Lehner, F. Pretorius, and R. DeVoe, gr-qc/0612100 (2006).

[24] B. Vaishnav, D. Shoemaker, and S. Larson, in Proceeding of the 6th Annual International LISA Symposium (2006).

[25] C. Cutler, T. A. Apostolatos, L. Bildsten, L. S. Finn, 
E. E. Flanagan, D. Kennefick, D. M. Markovic, A. Ori, E. Poisson, G. J. Sussman, et al., Phys. Rev. Lett. 70, 2984 (1993).

[26] C. Van Den Broeck, Class. and Quan. Grav. 23, L51 (2006).

[27] C. Van Den Broeck and A. S. Sengupta, Class. and Quan. Grav. 24, 1089 (2007).

[28] C. Van Den Broeck and A. S. Sengupta, Class. and Quan. Grav. 24, 155 (2007).

[29] J. M. Bowen and J. W. York, Phys. Rev. D 21, 2047 (1980).

[30] S. Brandt and B. Brügmann, Phys. Rev. Lett. 78, 3606 (1997).

[31] T. Nakamura, K. Oohara, and Y. Kojima, Prog. Theor. Phys. Suppl. 90, 1 (1987).

[32] M. Shibata and T. Nakamura, Phys. Rev. D 52, 5428 (1995).

[33] T. Baumgarte and S. Shapiro, Phys. Rev. D 59, 024007 (1999).

[34] B. Brügmann, J. A. González, M. Hannam, S. Husa, and U. Sperhake, gr-qc/0610128 (2006).

[35] J. R. van Meter, J. G. Baker, M. Koppitz, and D. Choi, Phys. Rev. D 73, 124011 (2006).

[36] M. Alcubierre, B. Brügmann, P. Diener, M. Koppitz, D. Pollney, E. Seidel, and R. Takahashi, Phys. Rev. D 67, 084023 (2003).
[37] M. Campanelli, C. O. Lousto, P. Marronetti, and Y. Zlochower, Phys. Rev. Lett. 96, 111101 (2006).

[38] J. Baker, M. Campanelli, and C. Lousto, Phys. Rev. D 65, 044001 (2002).

[39] M. Ansorg, B. Brügmann, and W. Tichy, Phys. Rev. D 70, 064011 (2004).

[40] S. Husa, I. Hinder, and C. Lechner, Computer Physics Communications 174, 983 (2006).

[41] E. Schnetter, S. Hawley, and I. Hawke, Class. Quant. Grav. 21, 1465 (2004).

[42] J. G. Baker, J. Centrella, D. Choi, M. Koppitz, and J. van Meter, Phys. Rev. D 73, 104002 (2006).

[43] G. B. Cook, Phys. Rev. D 50, 5025 (1994).

[44] T. W. Baumgarte, Phys. Rev. D 62, 024018 (2000).

[45] J. Baker, M. Campanelli, C. Lousto, and R. Takahashi, Phys. Rev. D 65, 124012 (2002).

[46] E. Berti, V. Cardoso, J. A. Gonzalez, U. Sperhake, M. Hannam, S. Husa, and B. Bruegmann, gr-qc/0703053 (2007).

[47] B. Owen, Phys. Rev. D 53, 6749 (1996).

[48] S. D. Mohanty, Phys. Rev. D 57, 630 (1998).

[49] B. S. Sathyaprakash and S. V. Dhurandhar, Phys. Rev. D 44, 3819 (1991).

[50] T. Damour, B. R. Iyer, and B. S. Sathyaprakash, Phys. Rev. D 57, 885 (1998). 